\title{
Effects of bradykinin on the survival of multiterritory perforator flaps in rats
}

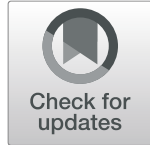

Jieke Wang ${ }^{\dagger}$, Encheng Ji ${ }^{\dagger}$, Chen Lin, Long Wang, Li Dai and Weiyang Gao*

\begin{abstract}
Background: Bradykinin, a vasoactive peptide, has many biological functions. For example, it accelerates angiogenesis. Thus, we studied the effects of bradykinin on the survival of perforator flaps.

Methods: Averagely, 50 male Sprague-Dawley rats were divided into control and bradykinin groups and underwent procedures to the multiterritory perforator flap. Areas of flap survival were tested 7 days later. Flap perfusion was evaluated by laser Doppler imaging. We assessed the extent of autophagy by determining LC3-II/I, Beclin 1, and p62. Flap angiogenesis was assessed by immunohistochemistry and H\&E staining. We measured the level of vascular endothelial growth factor (VEGF) protein using western blot. We assessed oxidative stress by measuring the activity of superoxide dismutase (SOD) and malondialdehyde (MDA) levels. The apoptotic index was also evaluated by western blot, and we determined nitric oxide (NO) production using an NO assay kit.
\end{abstract}

Results: The bradykinin group exhibited significantly larger areas of flap survival, higher blood supply, and more neovascularization. The bradykinin group also had higher SOD activity, higher VEGF expression and NO content, and reduced MDA compared to the control group. Rats treated with bradykinin also had lower levels of apoptosis and autophagy relative to the control group.

Conclusion: Our results suggest that bradykinin promotes the survival of multiterritory perforator flaps by increasing angiogenesis, promoting the release of $\mathrm{NO}$, suppressing apoptosis, reducing oxidative stress, and inhibiting autophagy.

Keywords: Bradykinin, Perforator flap, Apoptosis, Autophagy, Angiogenesis

\section{Background}

The skin, a protective barrier, is highly vulnerable to trauma which can cause serious skin defects. A multiterritory perforator flap can be used to cover these skin defects. It is a skin flap that involves a perforator artery of $0.5 \mathrm{~mm}$ or greater, and is widely used because of their advantages $[1,2]$. For example, when the skin is injured or defected by severe trauma and burn etc., the multiterritory perforator flaps can cover the huge skin defects. However, avoiding necrosis of such flaps remains a challenge. Many studies have proven that necrosis of the perforator flap always occurs at the dynamic and potential territories [3-5]. Previous studies have also demonstrated that inadequate

\footnotetext{
* Correspondence: weiyanggaoi@126.com

†Jieke Wang and Encheng Ji contributed equally to this work.

Department of Hand and Plastic Surgery, The Second Affiliated Hospital and

Yuying Children's Hospital of Wenzhou Medical University, The Second

School of Medicine, The Second Clinical Medical College of Wenzhou

Medical University, Wenzhou Medical University, No. 109, Xue Yuan Road (West), Lucheng District, Wenzhou 325000, China
}

blood supply [6], oxidative stress [7], and cells apoptosis [8] are important factors that lead to flap necrosis. Thus, there is a need to find an effective way to improve the survival of the multiterritory perforator flaps.

Bradykinin, a vasoactive peptide released from precursor kininogens by a protease known as kallikrein [9], is an important component of both acute and chronic inflammatory processes $[10,11]$. However, bradykinin performs several other biological functions as well. For example, it increases expression of VEGF via the bradykinin B2 receptor [12]. The levels of VEGF are particularly affected; vascular endothelial cells are stimulated to regenerate and proliferate, thus accelerating angiogenesis [13]. Yoshida et al. [14] recently reported that kallikrein gene delivery weakened apoptosis in ischemia-reperfusion (I/R) injury and myocardial infarction via bradykinin. Bradykinin had anti-apoptotic effects in a model of coronary artery disease [15]. Bradykinin also played an antioxidative role in a rat model of acute hyperglycemia [16], in which it reduced

(c) The Author(s). 2019 Open Access This article is distributed under the terms of the Creative Commons Attribution 4.0 International License (http://creativecommons.org/licenses/by/4.0/), which permits unrestricted use, distribution, and 
oxidative stress under conditions of hyperglycemia. Moreover, many studies suggest that bradykinin negatively regulates both apoptotic and autophagic responses via the PI3K/Akt signaling pathway [17-19]. Excessive autophagy is detrimental although the process of autophagy is advantageous to cell survival [20, 21]. Inhibition of autophagy could inhibit oxidative stress, suppress apoptosis, and enhance perforator flap survival [8].

Several recent publications have demonstrated that bradykinin stimulates nitric oxide (NO) production [22-24]. $\mathrm{NO}$, the product of nitric oxide synthase (NOS), promotes cell proliferation and angiogenesis [25]. NO also inhibits apoptosis and autophagy by stimulating the PI3K/Akt signaling pathway [19], which is beneficial for flap survival. Therefore, we hypothesized that bradykinin inhibits apoptosis and autophagy by promoting the release of NO, which may also improve flap survival.

The anti-apoptotic functions of bradykinin, with its ability to inhibit autophagy, inhibit oxidative stress, accelerate vascularization, and promote the release of NO, should be beneficial to improve multiterritory perforator flap survival. In this study, we hypothesized that bradykinin enhances the survival of multiterritory perforator flaps and analyzed its role in doing so in rats. We used histological and protein analyses to investigate whether bradykinin had these effects in a multiterritory perforator flap. We hope our study leads to novel strategies to improve flap survival.

\section{Methods}

\section{Animals}

Sprague-Dawley (SD) rats, with a closed group of genetic characteristics [26], were used in this study. Fifty healthy male specific-pathogen-free (SPF) rats weighing 250 to $300 \mathrm{~g}$ were purchased from the Wenzhou Medical University (license no. SCXK [ZJ] 2015-0001). All used procedures and animal care conformed to the Health Guidelines National Institutes for the Care and Use of Laboratory Animals. The Wenzhou Medical University Animal Research Committee approved the study (wydw 2014-0015). Rats were housed in separate cages with free access to food and water under standard environmental conditions such as temperature $22-25^{\circ} \mathrm{C}$, humidity $60-70 \%$, and 12-h light:12-h dark cycle. Rats were divided into two groups randomly: the bradykinin group and the saline (control) group. Each group contained 25 rats.

\section{Flap animal model}

Rats were anesthetized with intraperitoneal pentobarbital $(60 \mathrm{mg} / \mathrm{kg})$ [8]. Before surgery, we removed dorsal fur with a depilatory cream and electric shaver. A deep circumflex iliac artery (DCIA) flap was made on the right side of each rat dorsum [27]. In the particular flap, there are three vascular territories: the anatomic territory, the dynamic territory, and the potential territory. In this flap model, the anatomic territory has a deep circumflex iliac (DCI), the dynamic territory has an intercostal artery (IC), and the potential territory has a thoracodorsal artery (TD) [8]. The flap position was approximately $2.5 \times 11 \mathrm{~cm}$ in size, and it was standardized by bony landmarks. Transillumination identified the choke vessel zone (CVZ) between the IC and TD. Next, the flap was sutured into the original position after the TD and IC were ligated.

\section{Drug administration}

Rats in the bradykinin group were injected intraperitoneal bradykinin $(150 \mu \mathrm{g} / \mathrm{kg}$; purity $=98.97 \%$; Medchem Express, Princeton, NJ, USA) [28] $30 \mathrm{~min}$ before the procedure. Rats in the control group were injected normal saline at an equal volume for the same days. All rats were housed individually. All rats were sacrificed after 7 days.

\section{Flap assessment}

On postoperative day 7 (POD 7), we took high-resolution photographs of the flap with a digital camera. We measured surviving flap areas by superimposing the photographs onto graph paper. The percentages of the viable area were quantified as follows: (range of survival/ total flap size) $\times 100 \%$.

\section{Experimental design}

Five rats in each group were used for each test method. In details, five rats in each group were used for flap survival observation and Laser Doppler perfusion imaging; five rats in each group were used for hematoxylin and eosin (H\&E) and immunohistochemistry (IHC) staining; five rats in each group were used for superoxide dismutase (SOD) activity and malondialdehyde (MDA) content tests; five rats in each group were used for $\mathrm{NO}$ content test; and another five rats in each group were used for western blot.

\section{Hematoxylin and eosin staining}

A sample $(1 \times 1 \mathrm{~cm})$ from each flap CVZ [27] was collected after the sacrifice of rats on POD 7, and routine procedures of H\&E staining kit (Solarbio Science \& Technology, Beijing, China) were performed. The thickness of the flap tissue was measured under a light microscope, and the number of microvessels in each area $\left(/ \mathrm{mm}^{2}\right)$ was calculated to understand the condition of microvascular density (MVD).

\section{Western blot analysis}

On POD 7, flaps from the $\mathrm{CVZ}$ were stored at $-80^{\circ} \mathrm{C}$. We determined protein concentrations by using the BCA assay (Thermo Fisher Scientific, Rockford, IL, USA). We 
performed routine procedures. The membranes were incubated with the following primary antibodies: VEGF (1:1000; Abcam, Cambridge, UK), p62 (1:1000; Abcam, Cambridge, UK), Beclin 1 (1:1000; Cell Signaling Technology (CST), Danvers, MA, USA), LC3 (1:1000; Sigma-Aldrich, St. Louis, MO, USA), cleaved caspase-3 (1:1000; CST), Bax (1:1000; CST), BCL-2 (1:1000; CST) and GAPDH (1:2000; Bioworld Technology, St. Louis Park, MN, USA). Next, membranes were incubated with goat anti-rabbit secondary antibody for $2 \mathrm{~h}$. We quantified band intensity using Image Lab (ver. 5.2, Bio-Rad Laboratories, Hercules, CA, USA).

\section{Immunohistochemistry}

On POD 7, samples from the CVZ which were fixed in paraformaldehyde were sectioned into $5 \mu \mathrm{m}$ slices. Sections were rehydrated in a graded series of ethanol after they were deparaffinized through xylene. Then, sections were immersed in $3 \% \mathrm{H}_{2} \mathrm{O}_{2}$ and incubated to saturate nonspecific sites. Last sections were incubated with CD34 (1:50; Abcam, Cambridge, UK) at $4{ }^{\circ} \mathrm{C}$ overnight. Sections were imaged at $\times 100$ magnification on an image acquisition system (Olympus, Tokyo, Japan). The number of CD34-positive microvessels was calculated in five dense fields.

\section{Laser Doppler perfusion imaging}

On POD 7, blood perfusion images were obtained by a Laser Doppler instrument (Moor Instruments, Axminster, UK).

\section{Superoxide dismutase activity and malondialdehyde content}

SOD and MDA test kits (Nanjing Jiancheng Biology Institution, Nanjing, China) were used to measure the oxidative stress on the flaps. The flap specimens were obtained from the choke vessel zone on POD 7, weighed, homogenized, and diluted to $10 \%$ (volume/volume) on an ice bath. Then, SOD activity and MDA content were detected with the method as reported previously [27].

\section{NO content assay}

NO was assayed spectrophotometrically by measuring the products of NO metabolism using NO content assay kits [27] (Nanjing Jiancheng Biology Institution, Nanjing, China).

\section{Statistical analyses}

We performed statistical analyses using SPSS version 19.0 (SPSS, Chicago, IL, USA). All data are presented as means \pm standard errors of the mean (SEMs). We compared data between groups using Student's independent $t$ test and one-way repeated measures analysis of variance. In all analyses, $P<0.05$ was considered to indicate statistical significance.

\section{Results}

Surviving area and blood perfusion

The boundary between the surviving and necrotic regions was evident on POD 7 (Fig. 1a). The control group survival rate was $71.83 \pm 2.52 \%$, which differed significantly from that of the bradykinin group (85.83 \pm $0.98 \%)$. Compared to the control group, flap survival was better in the bradykinin group, with less necrosis $(P<0.01$; Fig. 1b). Laser Doppler images revealed the flap perfusion differences (perfusion units (PU)) were evident on POD 7 (Fig. 1c). Bradykinin improved blood supply at the CVZ compared to the control group (control group, $428.38 \pm 23.39$; bradykinin group, $505.85 \pm$ 25.52; $P<0.05$; Fig. 1d).

\section{Histology}

The flaps from rats treated with bradykinin showed more neovascularization and less necrosis than those from the control group (H\&E staining; Fig. 2a). The mean MVD in the CVZ was higher in flaps from the bradykinin group than the control group $(39.47 \pm 1.35$ vs. $30.38 \pm 2.10$, respectively; $P<0.05$; Fig. 2 b). Endothelial cells can be labeled by CD34. The number of CD34-positive vessels $/ \mathrm{mm}^{2}$ can indicate the mean MVD. Immunohistochemistry staining revealed that the number of CD34-positive vessels was higher in the bradykinin group than the control group $\left(42.13 \pm 2.59 / \mathrm{mm}^{2}\right.$ vs. $31.92 \pm 1.40 / \mathrm{mm}^{2}$, respectively; $P<0.05$; Fig. $\left.3 \mathrm{a}, \mathrm{b}\right)$.

\section{Western blot assay for VEGF}

The expression of VEGF in the CVZ of all perforator flaps was revealed by western blotting (Fig. 4a). VEGF expression was higher in the bradykinin group $(P<0.05$; Fig. 4b).

\section{Western blot analyses of the apoptotic index}

The expression of apoptotic proteins, including cleaved caspase-3, Bax, and Bcl-2, was investigated. Cleaved caspase- 3 and Bax are two types of apoptotic proteins that participate in apoptosis, whereas $\mathrm{Bcl}-2$ can resist apoptosis [20, 21]. The levels of cleaved caspase- 3 and Bax were decreased, whereas that of BCL-2 was increased on POD 7 in the bradykinin group $(P<0.05$; Fig. 5a, b).

\section{SOD and MDA content}

Compared to the control group, mean SOD activity was much higher in the bradykinin group $(45.46 \pm 1.43$ $\mathrm{U}$ mg protein ${ }^{-1}$ vs. $30.61 \pm 1.47 \mathrm{U} \mathrm{mg}$ protein $^{-1}$, respectively; $P<0.05$; Fig. $6 \mathrm{a}$ ). The bradykinin group also had a 
a

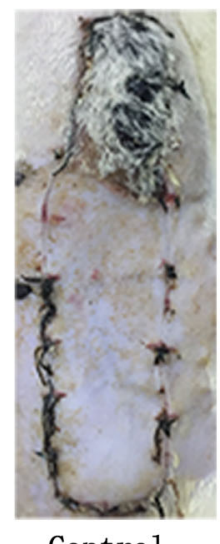

Control

$\mathrm{b}$

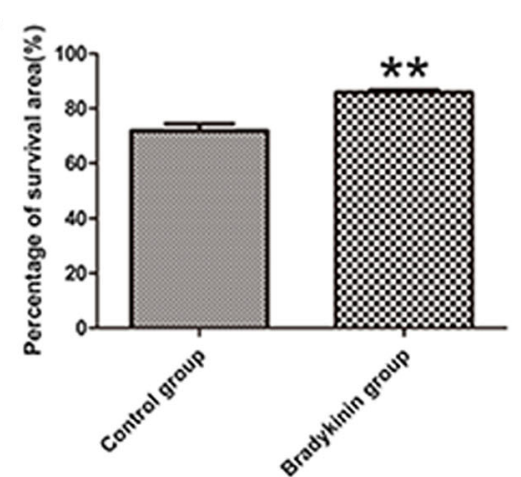

$\mathrm{c}$

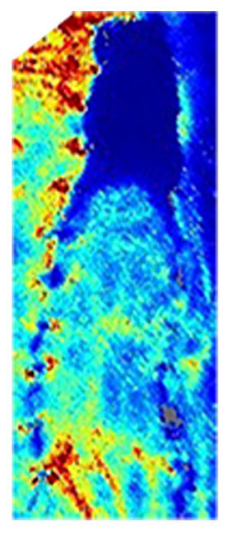

Control

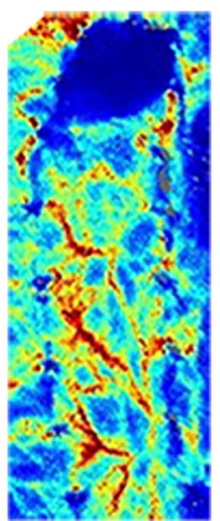

Bradykinin

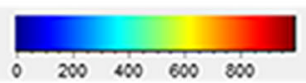

d

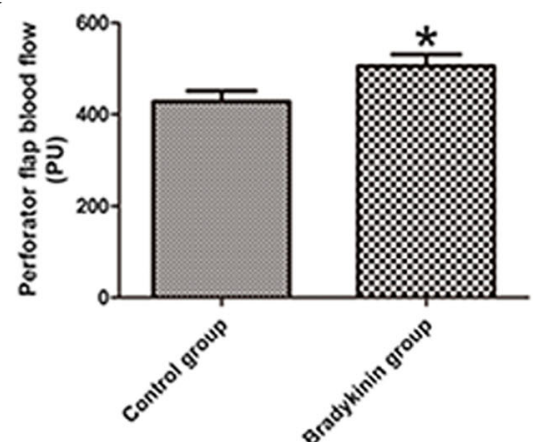

Fig. 1 a Photographs of the postoperative flaps from the bradykinin and control groups on day 7. b The flap survival rate (\%) in the bradykinin group $(85.83 \pm 0.98 \%)$ and control group $(71.83 \pm 2.52 \%)$. c The perfusion images of a flap on POD 7. Red denotes high perfusion, and blue denotes low perfusion with the scale bar. $\mathbf{d}$ The perfusion value on POD 7 (control group, 428.38 \pm 23.39 ; bradykinin group, $505.85 \pm 25.52$ ). $n=5$ per group. ${ }^{*} P<0.05,{ }^{* *} P<0.01$

much lower mean level of MDA $(41.39 \pm 1.67 \mathrm{nmol}$ mg protein $\left.^{-1}\right)\left(60.05 \pm 2.25 \mathrm{nmol} \mathrm{mg}\right.$ protein ${ }^{-1} ; P<0.05$; Fig. 6b).

\section{Western blot analyses of autophagy markers}

Western blot was used to assess Beclin 1 and LC3II/ LC3I expression in the CVZ of all perforator flaps. The ratio of LC3 II to LC3 I was downregulated as well as Beclin 1 in the bradykinin group. The control group had a higher LC3II/LC3I ratio and Beclin 1 expression than the bradykinin group $(P<0.05$; Fig. 7a). p62 is a marker of autophagic flux. Expression of p62 was much higher in the bradykinin group than the control group $(P<0.05$; Fig. 7b).

\section{NO content}

Compared to the control group, NO production was higher in the bradykinin group $(1.18 \pm 0.22$ vs. $0.82 \pm$ 0.15 , respectively; $P<0.05$; Fig. 8 ).

\section{Discussion}

Bradykinin, a vasoactive peptide, is produced by the protease degradation of high molecular weight or low molecular weight kininogens [29]. Bradykinin plays important roles in both acute and chronic inflammatory processes. However, it also has anti-apoptotic [30] and antioxidative [31] properties and promotes vascularization [32]. Thus, we hypothesized that bradykinin would improve the viability of multiterritory perforator flaps by promoting vascularization, suppressing apoptosis, inhibiting autophagy, promoting the release of NO, and reducing oxidative stress.

Several authors have reported that treatment with bradykinin increases VEGF expression in tumors [33]. VEGF can promote angiogenesis [13]. In a kininogendeficient rat tumor model (which cannot intrinsically generate bradykinin), expression of VEGF and the extent of angiogenesis were significantly less than in normal rats [34]. In skin flaps, VEGF which is particularly active in dermal vascular structures is secreted by fibroblasts and 


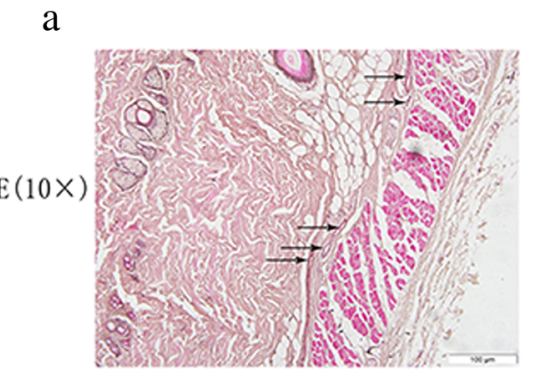

Control group

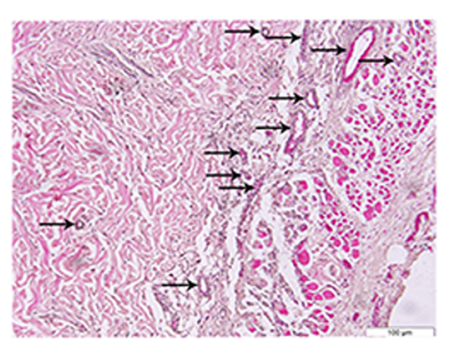

Bradykinin group

b

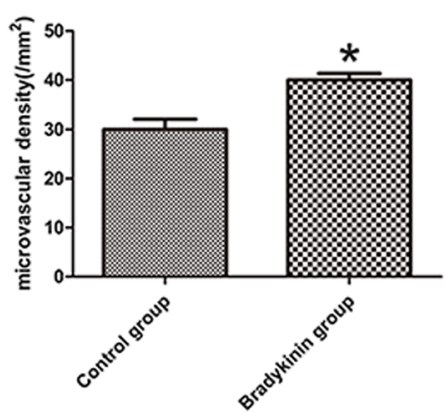

Fig. 2 a Neovascularization in the bradykinin and control groups (original magnification $\times 100$ ). $\mathbf{b}$ The percentage of microvascular density (MVD) in the bradykinin $\left(39.47 \pm 1.35 / \mathrm{mm}^{2}\right)$ and control $\left(30.38 \pm 2.10 / \mathrm{mm}^{2}\right)$ groups. $n=5$ per group. ${ }^{*} P<0.05$

keratinocytes in the cutis [35]. VEGF can also promote vascularization in multiterritory perforator flaps [27]. In our study, the VEGF protein levels were upregulated after treatment with bradykinin. Western blot analyses also showed lower VEGF levels in the control group than the bradykinin group. In addition, MVD showed more neovascularization in the bradykinin group relative to the control group. So, we know that bradykinin promotes vascularization in multiterritory perforator flaps by improving VEGF expression.

A postoperative flap that suffers from I/R injury exhibits increased production of ROS, apoptosis, inflammation,

\section{a}

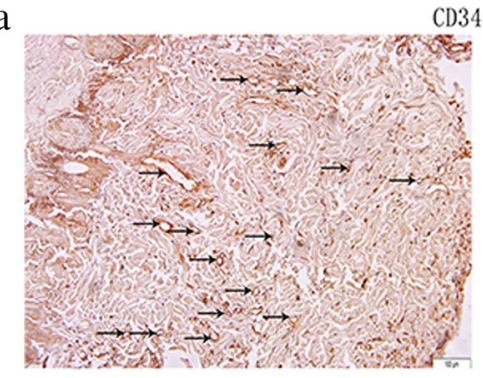

Control group
$\operatorname{CD} 34(10 \times)$

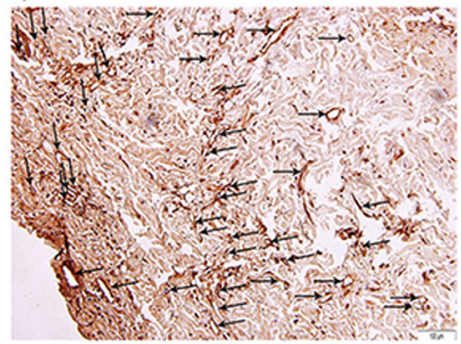

Bradykinin group

b

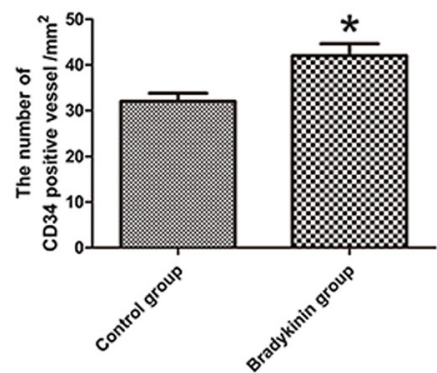

Fig. 3 a The CD34-positive microvessels were represented by black arrows (original magnification $\times 100$ ). $\mathbf{b}$ The number of CD34-positive vessels/ $\mathrm{mm}^{2}$ was $42.13 \pm 2.59 / \mathrm{mm}^{2}$ in the bradykinin group and $31.92 \pm 1.40 / \mathrm{mm}^{2}$ in the control group. $n=5$ per group. ${ }^{*} P<0.05$ 


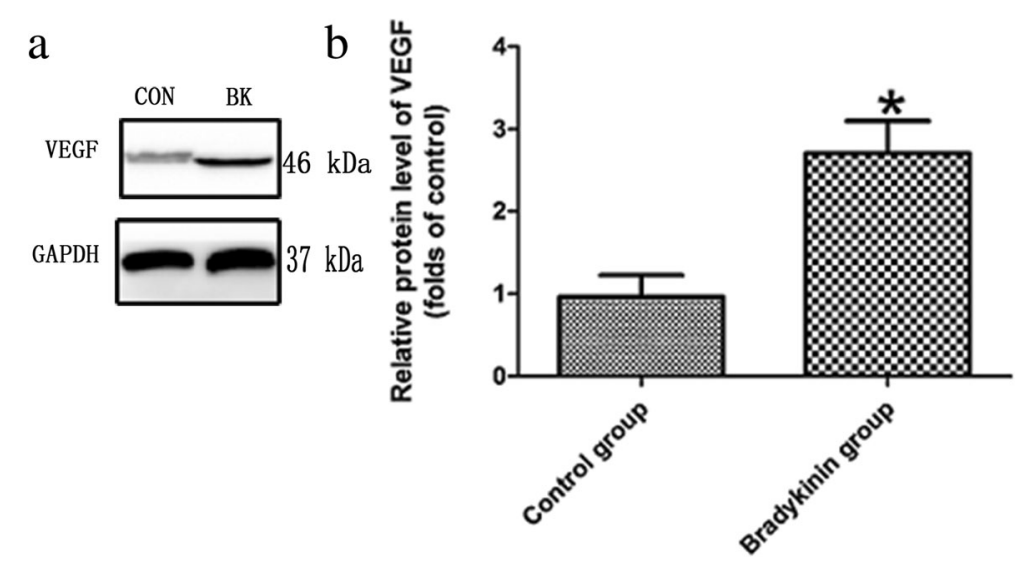

Fig. 4 a Levels of VEGF protein in the choke vessel zone (CVZ) were calculated as the fold relative to the control. GAPDH served as the loading control. $\mathbf{b}$ The level of VEGF. $n=5$ per group. ${ }^{*} p<0.05$

and so forth [36, 37]. Several publications have described effective therapeutic strategies for inhibiting $I / R$ injury [36-38]. Among them, the use of hyperbaric oxygen to inhibit I/R injury is accepted both clinically and experimentally [39, 40]. Flap survival is further improved by combining treatment with hyperbaric oxygen and vascular growth factor [41, 42]. As mentioned previously, the pathophysiological action of $I / R$ injury determined the final outcome of the flap.

Apoptosis plays a vital role in flap survival. The I/R process can induce apoptosis which can lead to cell death [43]. Burns et al. reported that cellular apoptosis can lead to I/R injury [43]. With less apoptosis, flaps exhibit a better blood supply and larger survival area [44]. When apoptosis is exacerbated, attenuation of apoptosis ameliorates healing. Bradykinin had an anti-apoptotic effect under conditions of diabetes [45]. Our results also show that bradykinin inhibits apoptosis, as reflected by increased BCL-2 levels and decreased Bax levels [46, 47]. We also found that cleaved caspase-3 activity was reduced in the bradykinin group [48]. So, we reach the conclusion that bradykinin can suppress apoptosis in a multiterritory perforator flap.

$\mathrm{I} / \mathrm{R}$ injury can produce ROS, a significant component of the complex oxidation process. ROS include free radicals, oxygen ions, and peroxides that initiate $\mathrm{I} / \mathrm{R}$ damage [49-51]. ROS formed during I/R injury cause a lot of changes that damage microcirculation (e.g., swelling of endothelial cells, vasoconstriction) [37]. When skin flaps experience ischemia, the oxidase system will be upregulated significantly $[52,53]$. This system is an important source of ROS production during ischemia [54, 55]. Inhibition of the xanthine oxidase system reduces the formation of ROS and increases the survival rate of flaps

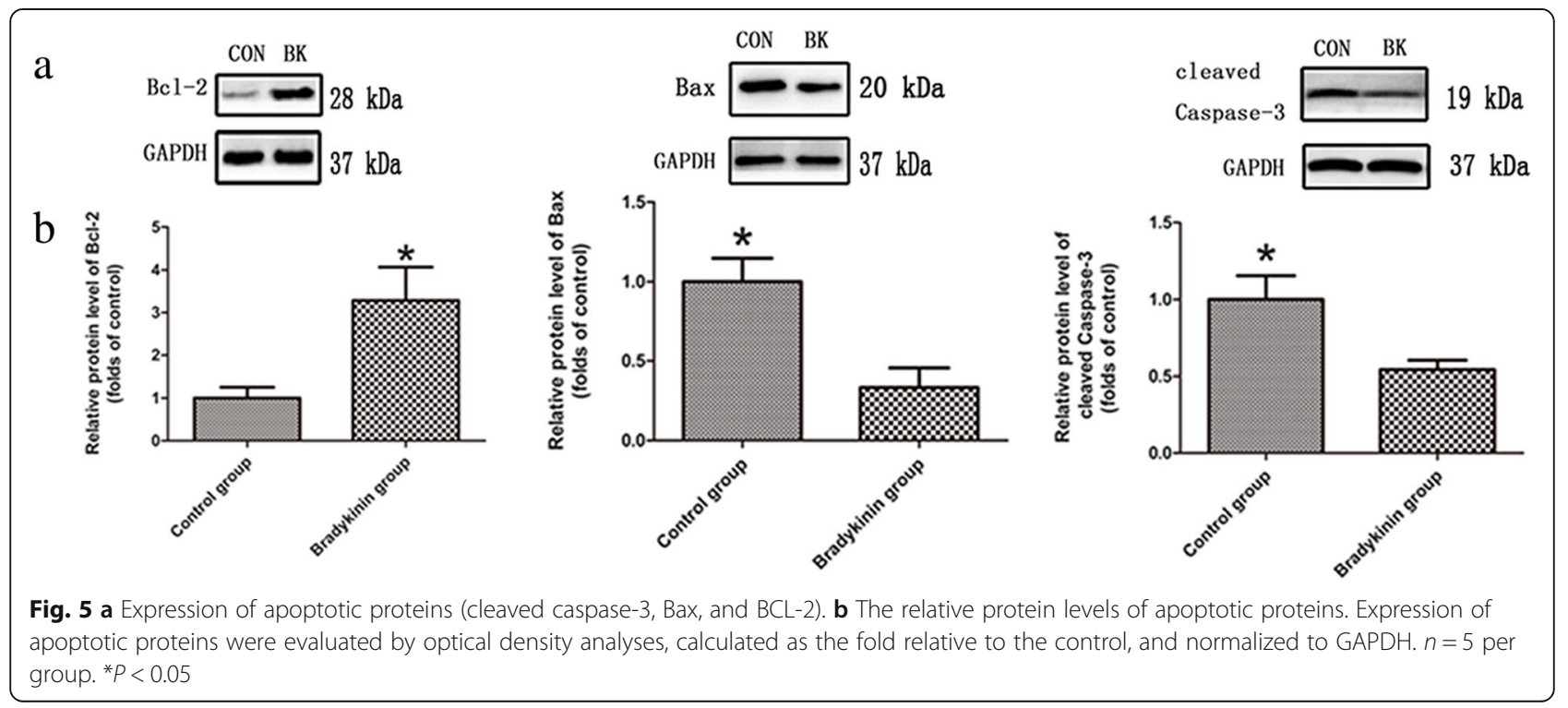



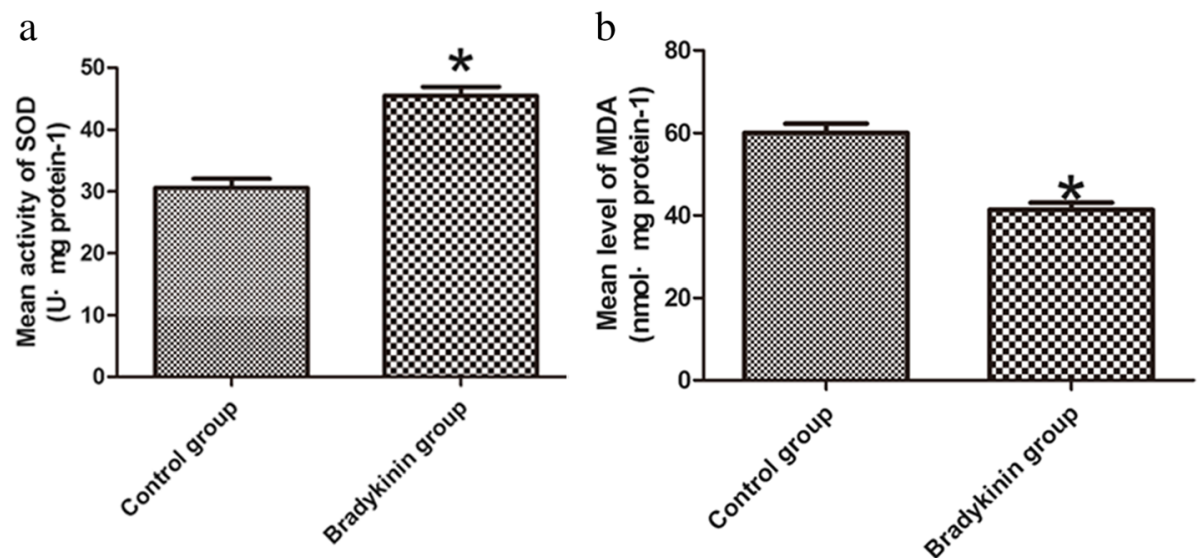

Fig. 6 a SOD activity $\left(U \mathrm{mg}^{-1}\right.$ protein $\left.{ }^{-1}\right)$. Bradykinin group, $45.46 \pm 1.43$; control group, $30.61 \pm 1.47$. b MDA content (nmol mg protein $\left.{ }^{-1}\right)$. Bradykinin group, $41.39 \pm 1.67$; control group, $60.05 \pm 2.25 . n=5$ per group. ${ }^{*} P<0.05$

$[53,56]$. MDA content and SOD activity are biomarkers of oxidative stress. In our research, we used MDA content and SOD activity to assess oxidative stress. Bradykinin has a cardioprotective effect in acute cardiac I/R injury [57]. In this research, the MDA content was much lower in the bradykinin group relative to the control group, whereas SOD activity was higher. Thus, bradykinin suppresses oxidative stress in multiterritory perforator flaps.

ROS can induce angiogenesis but an excessive amount of ROS inhibits angiogenesis [58-60]. It remains to be determined about the effects of ROS on angiogenesis. These effects are likely dependent on different characteristics of a disease. In the present study, bradykinin improved the survival of multiterritory perforator flaps by suppressing oxidative stress and accelerating vascularization. However, the relationship between oxidative stress and vascularization in multiterritory perforator flaps after treatment with bradykinin remains to be confirmed.

Scherz-Shouval and Elazar previously reported that ROS induces autophagy [61]. By contrast, Vande et al. reported that excessive autophagy increased the level of ROS production [62]. Excessive autophagic activity has a detrimental effect. It can consume functional components under conditions of excessive autophagy and promote cell death [20, 63]. For instance, many experts have reported that excessive autophagy enhances apoptosis, ROS production, and tissue injury [62-64]. The effects of autophagy vary by disease or different periods of a disease [65]. Wang et al. reported that inhibiting

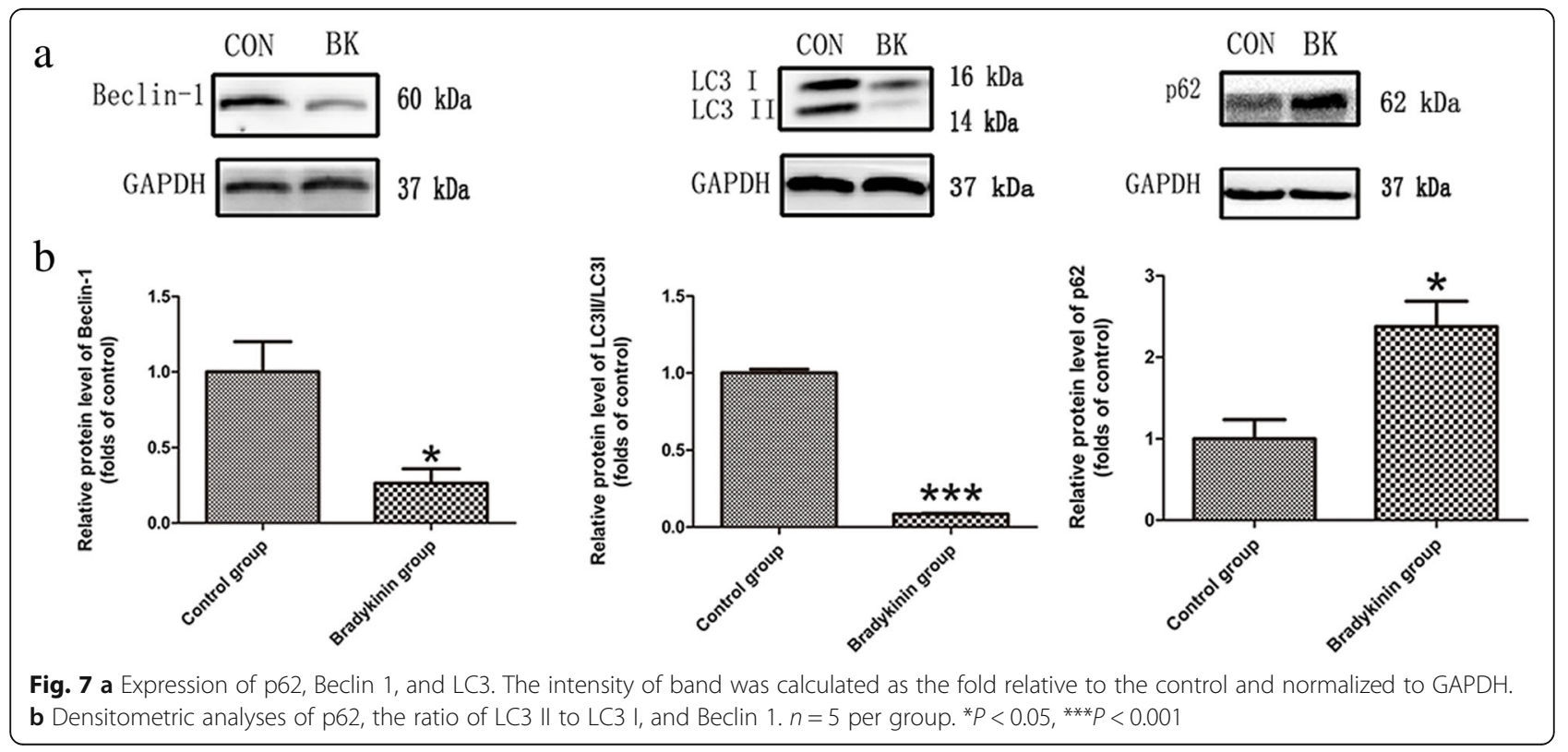




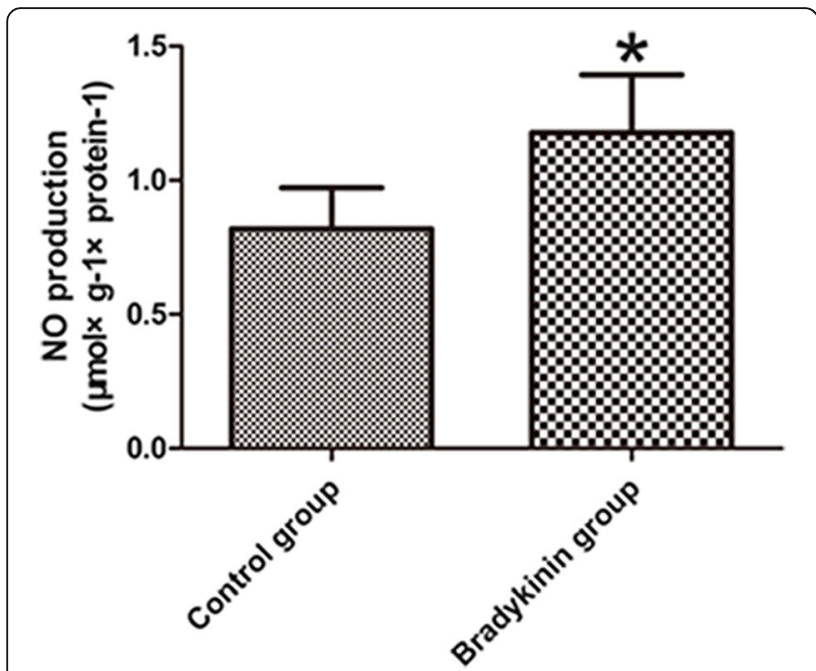

Fig. 8 Nitric oxide (NO) production in the CVZ on POD 7. $n=5$ per group. ${ }^{*} P<0.05$

autophagy enhanced perforator flap survival [8]. In the current study, bradykinin also regulated autophagy levels. Western blot analyses revealed that expression of LC3II/LC3I and Beclin 1 decreased, which indicates that autophagy was inhibited in the bradykinin group. p62 is an autophagic flux marker. p62 was increased in the group treated with bradykinin, which indicates that autophagy was inhibited. Thus, bradykinin likely downregulates the level of autophagy in multiterritory perforator flaps.

Several authors have reported that bradykinin can activate the PI3K/Akt signaling pathway $[18,24,66]$. This signaling pathway promotes the release of $\mathrm{NO}[22,23]$. Low levels of NO inhibit PTEN activity, thereby stimulating the Akt signaling pathway, which suppresses neuronal apoptosis [67]. Inactivation of PTEN results in increased mTORC1 activity, leading to the inhibition of autophagy [19]. NO, the product of NOS, promotes cell proliferation and angiogenesis [25], which is also beneficial for flap survival. In our study, NO levels decreased in the control group compared to the bradykinin group. Thus, NO inhibits apoptosis, autophagy, and tissue injuries. From the results, we conclude that bradykinin suppresses apoptosis and autophagy by promoting the release of NO in perforator flaps. The function of NO in angiogenesis is also important for flap survival.

\section{Conclusion}

Bradykinin increased angiogenesis, suppressed apoptosis, inhibited autophagy, and suppressed oxidative stress, leading to higher perforator flap survival rate. In addition, NO production was promoted in perforator flaps treated with bradykinin, which may suppress apoptosis and inhibit autophagy.

\section{Abbreviations}

BK: Bradykinin; CON: Control; CST: Cell Signaling Technology; CVZ: Choke vessel zone; DCl: Deep circumflex iliac; DCIA: Deep circumflex iliac artery; H\&E: Hematoxylin and eosin; I/R: Ischemia-reperfusion; IC: Intercostal artery; IHC: Immunohistochemistry; MDA: Malondialdehyde; MVD: Microvascular density; NO: Nitric oxide; NOS: Nitric oxide synthase; POD: Postoperative day; PU: Perfusion units; SEMs: Standard errors of the mean; SOD: Superoxide dismutase; TD: Thoracodorsal artery; VEGF: Vascular endothelial growth factor

\section{Acknowledgements}

Not applicable

\section{Funding}

This research was sustained by Zhejiang Province Medical Science and Technology Project of China (2014ZDA015) and Zhejiang Province Natural Science Foundation of China (LY15H060010).

\section{Availability of data and materials}

All data generated or analysed during this study are included in this published article [and its supplementary information files].

\section{Authors' contributions}

JW designed the study. CL, LW, LD, and JW collected the data. JW performed the statistical analysis. JW and EJ drafted the manuscript. JW and WG revised and proofread the manuscript. All authors read and approved the final manuscript.

\section{Ethics approval and consent to participate}

Eighty healthy male Sprague-Dawley rats weighing 250 to $300 \mathrm{~g}$ were purchased from Wenzhou Medical University (License No. SCXK [ZJ] 2015-0001). All use procedures and animal care conformed to the Health Guidelines National Institutes for the Care and Use of Laboratory Animals. The Wenzhou Medical University Animal Research Committee approved the study (wydw 2014-0015).

\section{Consent for publication}

Not applicable

\section{Competing interests}

The authors declare that they have no competing interests.

\section{Publisher's Note}

Springer Nature remains neutral with regard to jurisdictional claims in published maps and institutional affiliations.

Received: 8 November 2018 Accepted: 27 January 2019 Published online: 27 February 2019

\section{References}

1. Taylor Gl, Corlett RJ, Dhar SC, Ashton MW. The anatomical (angiosome) and clinical territories of cutaneous perforating arteries: development of the concept and designing safe flaps. Plast Reconstr Surg. 2011;127(4):1447-59.

2. Morris SF, Tang M, Almutari K, Geddes C, Yang D. The anatomic basis of perforator flaps. Clin Plast Surg. 2010;37(4):553-70 xi.

3. Taylor Gl, Corlett RJ, Caddy CM, Zelt RG. An anatomic review of the delay phenomenon: II. Clin Appl Plast Reconstr Surg. 1992;89(3):408-16 discussion 417-408.

4. Miyamoto S, Minabe T, Harii K. Effect of recipient arterial blood inflow on free flap survival area. Plast Reconstr Surg. 2008;121(2):505-13.

5. Fichter AM, Ritschl LM, Robitzky LK, et al. Impact of different antithrombotics on the microcirculation and viability of perforator-based ischaemic skin flaps in a small animal model. Sci Rep. 2016;6:35833.

6. Yang M, Sheng L, Li H, Weng R, Li QF. Improvement of the skin flap survival with the bone marrow-derived mononuclear cells transplantation in a rat model. Microsurgery. 2010;30(4):275-81.

7. Lima LP, de Oliveira AA, de Lima Silva JJ, et al. Electroacupuncture attenuates oxidative stress in random skin flaps in rats. Aesthet Plast Surg. 2012;36(5):1230-5.

8. Wang $L$, Jin Z, Wang J, et al. Detrimental effect of hypoxia-inducible factor1alpha-induced autophagy on multiterritory perforator flap survival in rats. Sci Rep. 2017;7(1):11791. 
9. Dewhirst MW, Vinuya RZ, Ong ET, et al. Effects of bradykinin on the hemodynamics of tumor and granulating normal tissue microvasculature. Radiat Res. 1992;130(3):345-54.

10. Steranka LR, Farmer SG, Burch RM. Antagonists of B2 bradykinin receptors. FASEB J. 1989;3(9):2019-25

11. Kaplan AP, Silverberg M, Ghebrehiwet B, Atkins P, Zweiman B. The kallikreinkinin system in inflammation. Adv Exp Med Biol. 1989;247A:125-36.

12. Miura S, Matsuo $Y$, Saku K. Transactivation of KDR/Flk-1 by the B2 receptor induces tube formation in human coronary endothelial cells. Hypertension. 2003;41(5):1118-23.

13. Kalka C, Tehrani H, Laudenberg B, et al. VEGF gene transfer mobilizes endothelial progenitor cells in patients with inoperable coronary disease. Ann Thorac Surg. 2000;70(3):829-34

14. Yoshida H, Zhang JJ, Chao L, Chao J. Kallikrein gene delivery attenuates myocardial infarction and apoptosis after myocardial ischemia and reperfusion. Hypertension. 2000;35(1 Pt 1):25-31.

15. Cangiano E, Marchesini J, Campo G, et al. ACE inhibition modulates endothelial apoptosis and renewal via endothelial progenitor cells in patients with acute coronary syndromes. Am J Cardiovasc Drugs. 2011;11(3): 189-98.

16. Mikrut K, Paluszak J, Kozlik J, et al. The effect of bradykinin on the oxidative state of rats with acute hyperglycaemia. Diabetes Res Clin Pract. 2001;51(2):79-85.

17. $X u X, T u L$, Jiang $W$, et al. Bradykinin prevents the apoptosis of NIT-1 cells induced by TNF-alpha via the PI3K/Akt and MAPK signaling pathways. Int J Mol Med. 2012;29(5):891-8.

18. Hammerschmidt S, Kuhn H, Gessner C, Seyfarth HJ, Wirtz H. Stretch-induced alveolar type II cell apoptosis: role of endogenous bradykinin and PI3K-Akt signaling. Am J Respir Cell Mol Biol. 2007;37(6):699-705.

19. Kaminskyy VO, Zhivotovsky B. Free radicals in cross talk between autophagy and apoptosis. Antioxid Redox Signal. 2014;21(1):86-102.

20. Smith CM, Chen Y, Sullivan ML, Kochanek PM, Clark RS. Autophagy in acute brain injury: feast, famine, or folly? Neurobiol Dis. 2011:43(1):52-9.

21. Nishida K, Yamaguchi O, Otsu K. Crosstalk between autophagy and apoptosis in heart disease. Circ Res. 2008;103(4):343-51.

22. Sheng ZL, Yao YY, Li YF, Fu C, Ma GS. Transplantation of bradykininpreconditioned human endothelial progenitor cells improves cardiac function via enhanced Akt/eNOS phosphorylation and angiogenesis. Am J Transl Res. 2015;7(7):1214-26.

23. Yang XM, Krieg T, Cui L, Downey JM, Cohen MV. NECA and bradykinin at reperfusion reduce infarction in rabbit hearts by signaling through $\mathrm{PI} 3 \mathrm{~K}$, ERK, and NO. J Mol Cell Cardiol. 2004;36(3):411-21.

24. Bell RM, Yellon DM. Bradykinin limits infarction when administered as an adjunct to reperfusion in mouse heart: the role of PI3K, Akt and eNOS. J Mol Cell Cardiol. 2003:35(2):185-93.

25. Moraes MS, Costa PE, Batista WL, et al. Endothelium-derived nitric oxide (NO) activates the NO-epidermal growth factor receptor-mediated signaling pathway in bradykinin-stimulated angiogenesis. Arch Biochem Biophys. 2014;558:14-27.

26. Malkesman $\mathrm{O}$, Braw $Y$, Maayan $\mathrm{R}$, et al. Two different putative genetic animal models of childhood depression. Biol Psychiatry. 2006:59(1):17-23.

27. Tao $X Y$, Wang $L, G a o W Y$, et al. The effect of inducible nitric oxide synthase on multiterritory perforator flap survival in rats. J Reconstr Microsurg. 2016; 32(9):643-9.

28. Nemethova M, Talian I, Danielisova V, et al. Delayed bradykinin postconditioning modulates intrinsic neuroprotective enzyme expression in the rat CA1 region after cerebral ischemia: a proteomic study. Metab Brain Dis. 2016;31(6):1391-403.

29. Deacon K, Knox AJ. Human airway smooth muscle cells secrete amphiregulin via bradykinin/COX-2/PGE2, inducing COX-2, CXCL8, and VEGF expression in airway epithelial cells. Am J Physiol Lung Cell Mol Physiol. 2015:309(3):L237-49.

30. Kono Y, Sawada S, Kawahara T, et al. Bradykinin inhibits serum-depletioninduced apoptosis of human vascular endothelial cells by inducing nitric oxide via calcium ion kinetics. J Cardiovasc Pharmacol. 2002;39(2):251-61.

31. Kayashima $Y$, Smithies $O$, Kakoki M. The kallikrein-kinin system and oxidative stress. Curr Opin Nephrol Hypertens. 2012;21(1):92-6.

32. Parenti A, Morbidelli L, Ledda F, Granger HJ, Ziche M. The bradykinin/B1 receptor promotes angiogenesis by up-regulation of endogenous FGF-2 in endothelium via the nitric oxide synthase pathway. FASEB J. 2001;15(8): 1487-9.
33. Ishihara K, Kamata M, Hayashi I, Yamashina S, Majima M. Roles of bradykinin in vascular permeability and angiogenesis in solid tumor. Int Immunopharmacol. 2002;2(4):499-509.

34. Ikeda Y, Hayashi I, Kamoshita E, et al. Host stromal bradykinin B2 receptor signaling facilitates tumor-associated angiogenesis and tumor growth. Cancer Res. 2004;64(15):5178-85.

35. Detmar M, Yeo KT, Nagy JA, et al. Keratinocyte-derived vascular permeability factor (vascular endothelial growth factor) is a potent mitogen for dermal microvascular endothelial cells. J Invest Dermatol. 1995;105(1):44-50.

36. Wang WZ, Baynosa RC, Zamboni WA. Update on ischemia-reperfusion injury for the plastic surgeon: 2011. Plast Reconstr Surg. 2011;128(6):685e-92e.

37. van den Heuvel MG, Buurman WA, Bast A, van der Hulst RR. Review: ischaemia-reperfusion injury in flap surgery. J Plast Reconstr Aesthet Surg. 2009;62(6):721-6.

38. Leite MT, Gomes HC, Percario S, Russo CR, Ferreira LM. Dimethyl sulfoxide as a block to the deleterious effect of nicotine in a random skin flap in the rat. Plast Reconstr Surg. 2007;120(7):1819-22.

39. Xiao YD, Liu YQ, Li JL, et al. Hyperbaric oxygen preconditioning inhibits skin flap apoptosis in a rat ischemia-reperfusion model. J Surg Res. 2015; 199(2):732-9.

40. Perrins DJ. Influence of hyperbaric oxygen on the survival of split skin grafts. Lancet. 1967;1(7495):868-71.

41. Bayati S, Russell RC, Roth AC. Stimulation of angiogenesis to improve the viability of prefabricated flaps. Plast Reconstr Surg. 1998;101(5):1290-5.

42. Xie XG, Zhang M, Dai YK, Ding MS, Meng SD. Combination of vascular endothelial growth factor-loaded microspheres and hyperbaric oxygen on random skin flap survival in rats. Exp Ther Med. 2015;10(3):954-8.

43. Burns AT, Davies DR, McLaren AJ, et al. Apoptosis in ischemia/reperfusion injury of human renal allografts. Transplantation. 1998;66(7):872-6.

44. Song $\mathrm{K}$, Zhang M, Hu J, et al. Methane-rich saline attenuates ischemia/ reperfusion injury of abdominal skin flaps in rats via regulating apoptosis level. BMC Surg. 2015:15:92.

45. Kwak SJ, Paeng J, Kim DH, et al. Local kallikrein-kinin system is involved in podocyte apoptosis under diabetic conditions. Apoptosis. 2011;16(5):478-90.

46. Xia CF, Yin H, Yao YY, et al. Kallikrein protects against ischemic stroke by inhibiting apoptosis and inflammation and promoting angiogenesis and neurogenesis. Hum Gene Ther. 2006;17(2):206-19.

47. Xia CF, Yin H, Borlongan CV, Chao L, Chao J. Kallikrein gene transfer protects against ischemic stroke by promoting glial cell migration and inhibiting apoptosis. Hypertension. 2004;43(2):452-9.

48. Tang $M$, Cui $M$, Dong $Q$, et al. The bradykinin B2 receptor mediates hypoxia/reoxygenation induced neuronal cell apoptosis through the ERK1/2 pathway. Neurosci Lett. 2009;450(1):40-4.

49. Kerrigan $\mathrm{CL}$, Stotland MA. Ischemia reperfusion injury: a review. Microsurgery. 1993;14(3):165-75.

50. Aydogan $\mathrm{H}$, Gurlek A, Parlakpinar $\mathrm{H}$, et al. Beneficial effects of caffeic acid phenethyl ester (CAPE) on the ischaemia-reperfusion injury in rat skin flaps. J Plast Reconstr Aesthet Surg. 2007;60(5):563-8.

51. Feng GM, Yang WG, Huan-Tang Chen $\mathrm{S}$, et al. Periodic alterations of jejunal mucosa morphology following free microvascular transfer for pharyngoesophageal reconstruction. J Plast Reconstr Aesthet Surg. 2006; 59(12):1312-7

52. Im MJ, Hoopes JE, Yoshimura Y, Manson PN, Bulkley GB. Xanthine: acceptor oxidoreductase activities in ischemic rat skin flaps. J Surg Res. 1989;46(3):230-4.

53. Angel MF, Im MJ, Chung HK, Vander Kolk CA, Manson PN. Effects of combined cold and hyperbaric oxygen storage on free flap survival. Microsurgery. 1994;15(9):648-51.

54. Shah AM, Channon KM. Free radicals and redox signalling in cardiovascular disease. Heart. 2004;90(5):486-7.

55. Kakita T, Suzuki M, Takeuchi H, Unno M, Matsuno S. Significance of xanthine oxidase in the production of intracellular oxygen radicals in an in-vitro hypoxia-reoxygenation model. J Hepato-Biliary-Pancreat Surg. 2002;9(2):249-55.

56. Im MJ, Manson PN, Bulkley GB, Hoopes JE. Effects of superoxide dismutase and allopurinol on the survival of acute island skin flaps. Ann Surg. 1985; 201(3):357-9.

57. Egom EE, Ke Y, Solaro RJ, Lei M. Cardioprotection in ischemia/reperfusion injury: spotlight on sphingosine-1-phosphate and bradykinin signalling. Prog Biophys Mol Biol. 2010;103(1):142-7.

58. Ushio-Fukai M, Tang Y, Fukai T, et al. Novel role of gp91(phox)-containing $\mathrm{NAD}(\mathrm{P}) \mathrm{H}$ oxidase in vascular endothelial growth factor-induced signaling and angiogenesis. Circ Res. 2002;91(12):1160-7. 
59. West XZ, Malinin NL, Merkulova AA, et al. Oxidative stress induces angiogenesis by activating TLR2 with novel endogenous ligands. Nature. 2010;467(7318):972-6.

60. Okuno Y, Nakamura-Ishizu A, Otsu K, Suda T, Kubota Y. Pathological neoangiogenesis depends on oxidative stress regulation by ATM. Nat Med. 2012;18(8):1208-16.

61. Scherz-Shouval R, Elazar Z. Regulation of autophagy by ROS: physiology and pathology. Trends Biochem Sci. 2011;36(1):30-8.

62. Vande Velde C, Cizeau J, Dubik D, et al. BNIP3 and genetic control of necrosis-like cell death through the mitochondrial permeability transition pore. Mol Cell Biol. 2000;20(15):5454-68.

63. Matsui Y, Takagi H, Qu X, et al. Distinct roles of autophagy in the heart during ischemia and reperfusion: roles of AMP-activated protein kinase and Beclin 1 in mediating autophagy. Circ Res. 2007;100(6):914-22.

64. Zheng B, Mao JH, Oian L, et al. Pre-clinical evaluation of AZD-2014, a novel mTORC1/2 dual inhibitor, against renal cell carcinoma. Cancer Lett. 2015; 357(2):468-75.

65. Zhou KL, Zhang YH, Lin DS, Tao XY, Xu HZ. Effects of calcitriol on random skin flap survival in rats. Sci Rep. 2016;6:18945.

66. Dong R, Chen W, Feng W, et al. Exogenous bradykinin inhibits tissue factor induction and deep vein thrombosis via activating the eNOS/ phosphoinositide 3-kinase/Akt signaling pathway. Cell Physiol Biochem. 2015;37(4):1592-606

67. Numajiri N, Takasawa K, Nishiya T, et al. On-off system for PI3-kinase-Akt signaling through S-nitrosylation of phosphatase with sequence homology to tensin (PTEN). Proc Natl Acad Sci U S A. 2011;108(25):10349-54.

Ready to submit your research? Choose BMC and benefit from:

- fast, convenient online submission

- thorough peer review by experienced researchers in your field

- rapid publication on acceptance

- support for research data, including large and complex data types

- gold Open Access which fosters wider collaboration and increased citations

- maximum visibility for your research: over $100 \mathrm{M}$ website views per year

At $\mathrm{BMC}$, research is always in progress.

Learn more biomedcentral.com/submissions 